SCIENTIFIC LETTER

\title{
Prognostic value of baseline white blood cell count in patients with acute myocardial infarction and ST segment elevation
}

\author{
J, E Núñez, E Núñez, V Bertomeu, L Fácila, J Sanchis, V Bodí, R Sanjuán, M L Blasco, Á Martínez, \\ Á Llâcer
}

l: the setting of an acute myocardial infarction (AMI), elevated white blood cell count (WBC-C) has been associated with adverse cardiovascular events ${ }^{1-3}$ implying not only a purely reparative physiological role, but a pathologic one. Therefore, WBC-C has recently drawn the attention of researchers as a potential stratification tool because of its simplicity, cost effectiveness, and wide availability. The present study was designed to determine the independent predictive value of WBC-C on mortality at 30 days and one year after ST segment elevation acute myocardial infarction (STEMI).

\section{METHODS}

We studied a cohort of 515 consecutive patients admitted to our centre with STEMI from October 2000 to February 2003. STEMI was defined based on the criteria established by the American College of Cardiology and European Society of Cardiology. Total WBC-C $\left(\times 10^{9} / 1\right)$ was obtained in the emergency department within the first 24 hours of symptom onset. The treatment allocated to each patient, was individualised following established guidelines. The decision for surgical or percutaneous revascularisation was guided by the presence of ominous symptoms, like recurrent angina, severe heart failure, or a positive stress test at discharge. Death for any cause was selected as a main end point and ascertained either during hospitalisation, contacting patient/family by telephone, or by routine clinic visits.

Multiple linear regression analysis was used to explore the degree of association between WBC-C and those variables that traditionally have been associated with poor prognosis after STEMI. Cox regression analysis was used to calculate the crude, as well as the adjusted, incidence rates and hazard ratios (HR) with their respective 95\% confidence intervals (95\% CI).

The linearity assumption for continuous variables was assessed with proportional hazards additive model (GAM). In the absence of known biological evidence supporting a particular threshold along the continuum in the WBC-C, the GAM plot was also used as a criterion for variable categorisation.

\section{RESULTS}

At a median follow up of one year (interquartile range (IQ) range $=0.6$ ), a total of 99 deaths were identified; the proportion of deaths across the WBC-C increases steadily at 30 days as well as at one year. The univariate and multivariate adjusted risk trajectory between WBC-C and the $\log$ hazard ratio is depicted in fig 1. In multivariate analysis the association is monotonically positive until the $10 \times 10^{9} / 1$ point after which the relation becomes flat (fig 1B). For further analysis, WBC-C was categorised as follows: WBC-1, < $10 \times 10^{9} / \mathrm{l}$; WBC-2, $10-15 \times 10^{9} / \mathrm{l}$; and WBC-3, > $15 \times 10^{9} /$ l. In multivariate analysis (adjusted by
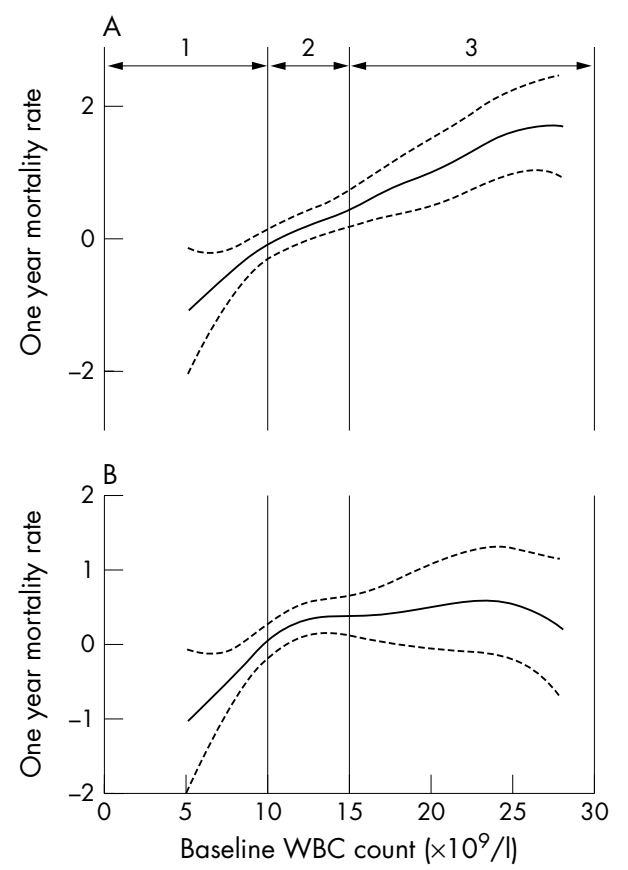

Figure 1 GAM plot characterising the relation between mortality rate and each value point in the continuum of WBC-C. Dash lines are $95 \% \mathrm{Cl}$. Numbers 1, 2, and 3 represent the stratums created when categorising WBC-C. (A) Univariate analysis. (B) Analysis adjusted by age, sex, Killip class, systolic blood pressure, reperfusion, new onset bundle branch block, and serum creatinine.

age, sex, Killip class, new onset bundle branch block, clinical evidence of reperfusion, systolic blood pressure, and serum creatinine) WBC-2 was associated with a twofold increase in the incidence of death at 30 days (HR 2.21, 95\% CI 1.13 to 4.31 ) and one year (HR 2.16, 95\% CI 1.27 to 3.7) compared with WBC-1. A similar gradient of risk was found between WBC-3 $v$ WBC- 1 at 30 days (HR 2.01, 95\% CI 0.90 to 4.46) and one year (HR 2.33, 95\% CI 1.21 to 4.48). A significant difference was found at one year $(0.862 v 0.853 ; \mathrm{p}=0.034)$ between the $\mathrm{C}$ statistics of the models with and without WBC-C.

Killip classification ranked as the most important variable associated with WBC-C, explaining up to $44.3 \%$ of the model predictability $(\mathrm{r}=0.28, \mathrm{p}<0.001)$. This finding highlighted the close relation between the leucocyte's initial response after STEMI and the degree of left ventricular dysfunction. On the other hand, the degree of association between WBC-C with troponin $(r=0.19, \mathrm{p}<0.001)$ or left ventricular ejection fraction $(\mathrm{r}=-0.15, \mathrm{p}=0.005)$ was less pronounced. 


\section{DISCUSSION}

In the present study, we demonstrated that WBC-C taken during the first 24 hours after admission, in patients with STEMI, was strongly and independently associated with 30 day and one year mortality. Furthermore, our results show that the gradient of risk flattened after the $10 \times 10^{9} / 1$ threshold, which in our cohort discriminates subjects with and without risk for future events, leading us to suggest, for sake of simplicity, the use of WBC-C binary as a prognostic factor.

Several mechanisms have been proposed to account for the association between WBC-C and mortality. The leucocyte response that occurred after STEMI is a central component of the systemic inflammatory response to injury and the reparative mechanism which is mounted in order to initiate the replacement of the infarct area by collagen. It follows that the greater the area of the myocardial necrosis, the greater the leucocyte response at systemic and local levels. ${ }^{45}$

On clinical grounds, we can only indirectly measure the extension of the infarction, by its effects on clinical indices such as peak concentrations of myocardial enzymes, left ventricular ejection fraction, or Killip class, all variables with established prognostic implications after STEMI. Killip class was shown to be the strongest and independent predictor for WBC-C, supporting the role of WBC-C as a surrogate for infarct extension. However, the fact that WBC-C has been shown to predict mortality independently of infarct size related variables ${ }^{12}$ it provides indirect evidence for an additional role in the pathogenesis of the post-AMI complications.

Based on the present study, WBC-C independently increased the risk of death at 30 days and one year in patients with STEMI. The gradient of risk starts at $10 \times 10^{9} / 1$ with no further increment beyond this point. More research on experimental grounds needs to be done to determine the precise role of leucocytosis in the cascade of pathophysiological events that occur during the ischaemic insult, and consequently, opening new therapeutic avenues designed to minimise further myocardial damage.

\section{Authors' affiliations}

J E Núñez, V Bertomeu, L Fácila, J Sanchis, V Bodí, Á Martínez,

Á Llâcer, Servicio de Cardiología, Hospital Clínic Universitari, University of Valencia, Valencia, Spain

E Núñez, University of Minnesota, Minneapolis, USA

R Sanjuán, M L Blasco, Unidad Coronaria. Hospital Clínic Universitari, University of Valencia

This paper was supported by a grant (RECAVA-FIS)

Correspondence to: Julio E Núñez, MD, Servicio de Cardiología. Hospital Clínico Universitario, Avda. Blasco lbáñez 17. 46010 Valencia, Spain; julionv@terra.es

Accepted 17 September 2004

\section{REFERENCES}

1 Barron HV, Cannon CP, Murphy SA, et al. Association between white blood cell count, epicardial blood flow, myocardial perfusion, and clinical outcomes in the setting of acute myocardial infarction: a thrombolysis in myocardial infarction 10 substudy. Circulation 2000;102:2329-34.

2 Barron HV, Harr SD, Radford MJ, et al. The association between white blood cell count and acute myocardial infarction mortality in patients $>$ or $=65$ years of age: findings from the cooperative cardiovascular project. J Am Coll Cardiol 2001;38:1654-61.

3 Furman MI, Gore JM, Anderson FA, et al. Elevated leukocyte count and adverse hospital events in patients with acute coronary syndromes: findings from the global registry of acute coronary events (GRACE). Am Heart $J$ 2004; 147:42-8.

4 Lucchesi BR. Modulation of leukocyte-mediated myocardial reperfusion injury. Annu Rev Physiol 1990;52:561-76.

5 Chatelain P, Latour JG, Tran D, et al. Neutrophil accumulation in experimental myocardial infarcts: relation with extent of injury and effect of reperfusion. Circulation 1987;75:1083-90.

\section{FROM BMJ JOURNALS \\ Residence in mountainous compared with lowland areas in relation to total and coronary mortality. A study in rural Greece}

Please visit the Heart website [www.heartjinl. com] for a link to the full text of this article.
Nikos Baibas, Antonia Trichopoulou, Eftihios Voridis, Dimitrios Trichopoulos

Study objective: To investigate the association of residence in mountainous or lowland areas with total and coronary mortality, in a cohort with 15 years of follow up.

Design and setting: Prospective study, based on the adult population of two lowland and one mountainous village in rural Greece. Baseline measurements on sociodemographic, lifestyle, somatometric, clinical, and biochemical variables were recorded in 1981 during a health survey and total and coronary deaths were ascertained on the basis of death certificates up to 1996.

Participants: 1198 men and women, who had participated in the 1981 survey. Analyses are based on 504 men and 646 women with complete data.

Main results: There were 150 deaths among men and 140 among women (coronary deaths: 34 and 33 respectively). In multivariate analysis with Cox regression, after adjustment for age, education, body weight, smoking, alcohol consumption, systolic blood pressure, serum total cholesterol, blood glucose, serum triglycerides, and serum uric acid, total and coronary mortality were lower for residents of the mountainous village in comparison with residents of the lowland villages (hazard ratios (95\% confidence intervals) for men and women, respectively: total mortality, 0.57 (0.38 to 0.84$)$ and 0.69 (0.47 to 1.02$)$; coronary mortality, 0.39 ( 0.16 to 0.98 ) and 0.46 ( 0.20 to 1.05$)$ ).

Conclusions: Residence in mountainous areas seems to have a "protective effect" from total and coronary mortality. Increased physical activity from walking on rugged terrains under conditions of moderate hypoxia among the mountain residents could explain these findings.

A Journal of Epidemiology and Community Health 2005;59:274-278. 